\title{
Design of Manchu Image Acquisition System Based on STM32
}

\author{
HongBo Hao ${ }^{1, *}$, Fulin Zheng ${ }^{2}$ \\ ${ }^{1}$ Tianjin University of Science and Technology, China; \\ ${ }^{2}$ Dalian Minzu University, Liaoning,China \\ E-mail: 2844011096@qq.com \\ www.tust.edu.cn
}

\begin{abstract}
This paper designs a Manchu image acquisition system based on STM32 to protect and utilize Manchu ancient books. The system uses $S T M 32 F 407$ as the core controller, with the help of image sensor, $L C D$ screen, and $S D$ storage module to complete the function of image acquisition, storage and upload of Manchu ancient books. This paper describes in detail the hardware circuit design, software programming and overall function debugging of the image acquisition system. In order to achieve the purpose of digital protection of Manchu ancient books, we can collect, display, save and upload images in different situations.
\end{abstract}

Keywords: Manchu, STM32F407, SD storage module, image acquisition system

\section{Introduction}

Manchu is also used to write Manchu characters. Manchu not only created Manchu characters, but also used Manchu characters to record a large number of customs historical stories. Therefore, Manchu has a very important position in the history of Chinese characters. Manchu ancient books also have important historical and cultural value. With the change of time, the preservation of Manchu ancient books is becoming more and more difficult and the researchers' research is more and more inconvenient. In order to facilitate the research of researchers, at the same time better preservation of Manchu ancient books, with the development of time, we need to use more advanced technology to protect ancient books. Considering the present situation of science and technology development, design cost and people's use mode, the first choice of literature protection method is to digitize ancient books. In this paper, we choose the Manchu image acquisition system based on STM32 to carry out the research. That is to use the ancient books digital technology to digitize the Manchu ancient books, so as to achieve the purpose of protecting and using the ancient books ${ }^{1}$.

(C) The 2021 International Conference on Artificial Life and Robotics (ICAROB2021), January 21 to 24, 2021
For the protection and utilization of Manchu ancient books to make a certain contribution. The research method of this topic can be applied to more ancient books protection work. After the digital processing of ancient books, it can save a lot of paper resources, reduce the generation of waste and reduce the felling of trees. It plays a certain role in protecting the environment. At the same time, digital preservation can save a lot of human resources, and the transmission speed is faster, realizing the coordinated development of economy, environment and society. Based on the embedded processor platform, using STM32 core controller and CMOS camera, this paper designs an image acquisition device to realize the acquisition, storage and display of Manchu images in ancient books.

\section{Overall scheme design}

In this paper, STM32F407 chip is used as the core controller of Manchu image acquisition system based on STM32. Image acquisition module, image saving module, image display module and key module are controlled by different $I O$ ports to realize image acquisition, storage 
display. At the same time, the data transmission module completes the connection with the upper computer.

According to the designed system scheme, the camera image acquisition module, image storage module, key control module and TFT liquid crystal display module are designed and built. After completing the circuit design, according to the relevant theory of image acquisition and processing and the camera control method, the system software design and overall debugging are completed. The function of collecting, displaying, saving and uploading Manchu images in ancient books is realized through the key control module ${ }^{2}$.

\section{Hardware circuit design}

\subsection{Design of core controller}

In this paper, the core controller is used to receive the images collected by the image sensor and transmit them to TFTLCD for display. According to the research requirements, STM32 series chips are selected. STM32F407 is selected as the core controller to complete the design. STM32F407 has a great improvement compared with STM32F1 series. First of all, the speed of $F S M C$ is very fast. The screen swiping speed of $F 407$ can reach $3000 \mathrm{~W}$ pixels per second, while $F 103$ is only about $500 \mathrm{~W}$. When using the image sensor with $200 \mathrm{~W}$ pixels, $F 103$ has a stuck phenomenon, and F407 can be perfectly processed.

\subsection{Touch the capacitor button}

This design uses the capacitive touch screen to complete, so we plan to use a capacitive touch button as the return key of the system. When the system enters a function module, touch the button to return to the upper level. The circuit diagram is shown in the Fig. 1 below.

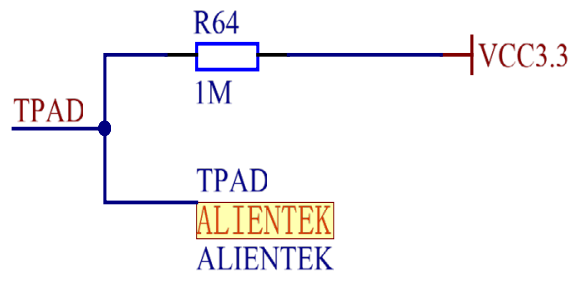

Fig.1. Capacitive touch button path diagram

\subsection{Image acquisition module}

In this paper, through the analysis and comparison of the performance of a variety of image acquisition sensors, for $C C D$ sensor, the photoelectric conversion of CMOS sensor is very direct and the efficiency is higher. With the development of microelectronic technology, the quality of $C M O S$ imaging is getting better and better, and the cost is lower than $C C D$. To sum up, this design uses $C M O S$ camera as the image sensor of this study.Through the study and research of CMOS image sensor, the circuit diagram design of image acquisition module is completed according to the function of different interfaces, as is shown in Fig. 2 below.

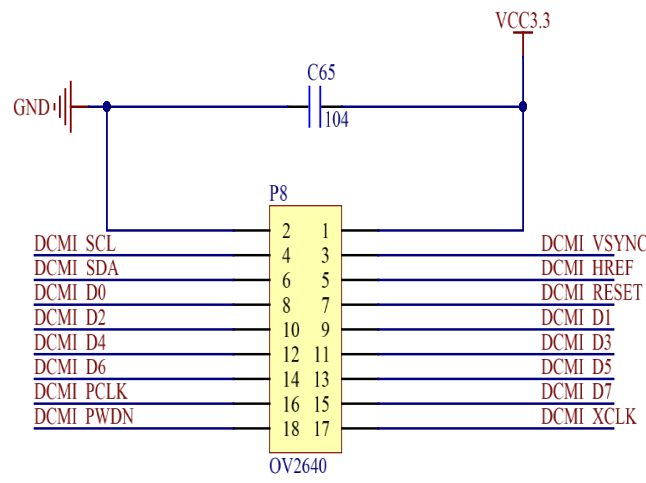

Fig.2. Image acquisition module circuit

In the interface of capacitive touch screen, $C S$ is the chip selection signal pin, which is effective at low level. $R S$ is the data command signal pin, 0 is the data and 1 is the command. $W R$ is the write enable signal, which is valid at low level. $R d$ can read the pin effectively. $R S T$ is a reset signal, which can control the reset of capacitive touch screen. $C S$ is the reset signal of capacitive touch screen. According to the function of each pin, the capacitor touch screen can be controlled to complete the required operation ${ }^{3}$.

\subsection{Storage module}

The research content of this paper is Manchu image acquisition, so the preservation of collected image is very important. After comparison and research, this paper uses an $8 G S D$ card as the storage module of Manchu image acquisition system. The circuit diagram is shown in Fig.3.

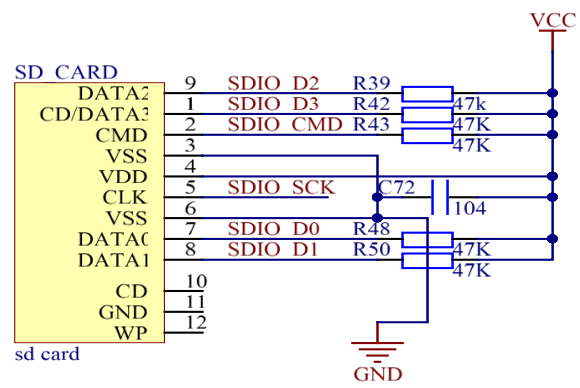

Fig.3. Circuit diagram of image storage module

As shown in the Fig.3, the $S D$ card has 6 communication lines and 3 power lines. The $C M D$ is a 
command line and a two-way signal line. DAT0, DAT1, $D A T 2$ and DAT3 are four data lines, which are also bidirectional signal lines. The transmission rate of each data line is 25 Mbit per second. The maximum is that four data lines read data at the same time, and the maximum can reach 100 Mbit

\subsection{Data transmission module}

The data transmission of this design is to transmit the collected image to the upper computer for saving or browsing. After comparing the speed and stability of serial communication, wireless communication and USB communication, USB communication is selected as the data transmission mode between Manchu image acquisition system and upper computer. USB transmission speed is faster and more stable, when transferring large files such as pictures, it is faster than serial transmission and Bluetooth transmission. WiFi transmission needs to install $W i F i$ module and specific host computer receiving. USB transmission does not need a specific host computer software. It can be convenient for data transmission with most computers. The standard of $U S B$ is four buses, VCC and GND, and $D+$ and $D$-. Because USB cannot be directly connected with STM32, the circuit diagram of this design is shown in Fig.4 below 4

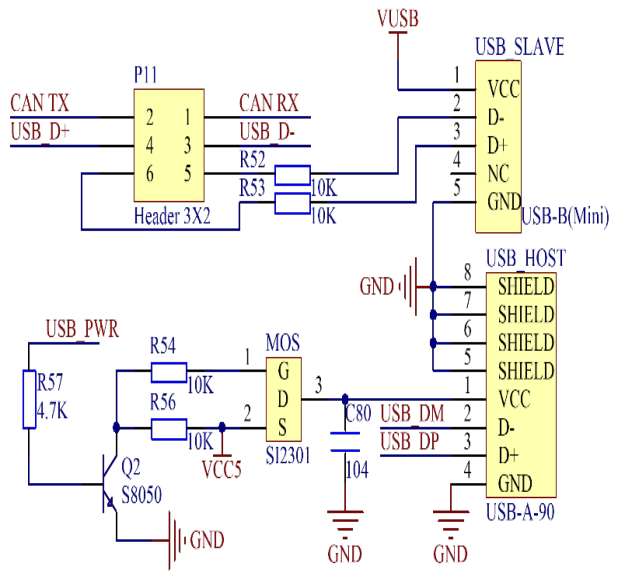

Fig.4. USB transmission circuit diagram

\section{Software design}

\subsection{Main program flow chart}

The program design of this chapter is based on Keil software and $\mathrm{C}$ language. Finally, the program design of Manchu image acquisition system is completed. In the main. C main program, the system initialization is to create a system_ Int function ${ }^{5}$. The flow chart of the whole is shown in Fig. 5 below.

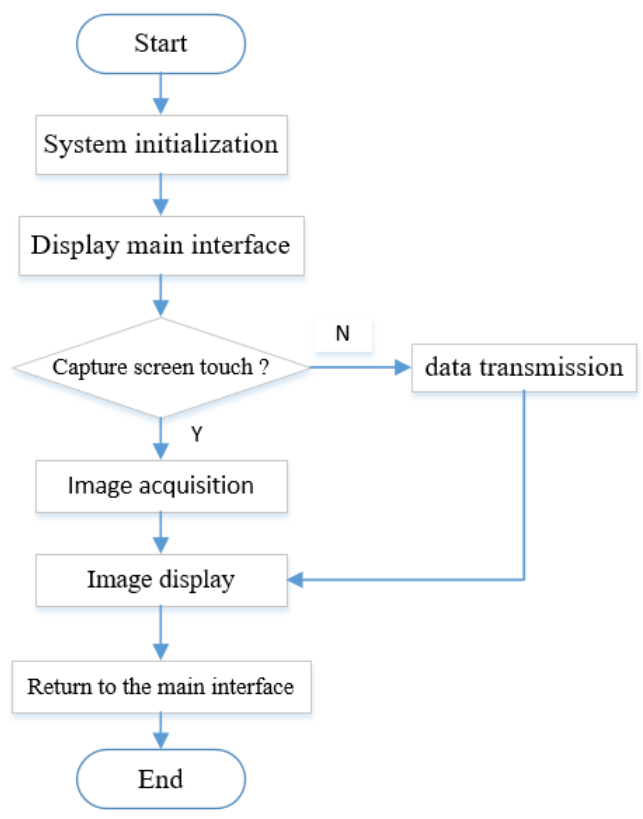

Fig.5. Main program flow chart

\subsection{Main program flow chart}

After the initialization, the initial interface is displayed. The initial interface mainly shows the English name of the research content in this paper and the detection results of whether each module is normal. It includes whether the icon image of $A P P$ exists, whether the screen is normal. The main interface is designed to display three main function icons, which are image acquisition, image display and USB transmission. Then the system detects whether there are touch screen events. If the three icons click events, it will enter different modules according to the click events.

\section{Conclusion}

\subsection{System}

There are many problems in the testing process of image acquisition. In the first acquisition, OV7725 image acquisition sensor is used, which is a $30 \mathrm{~W}$ pixel sensor. It is found that the collected images cannot be displayed clearly. After the teacher's help, the OV2640 image sensor was re selected to complete the image acquisition. 
It is the acquisition interface of Manchu image acquisition system, as is shown in the Fig. 6 below.

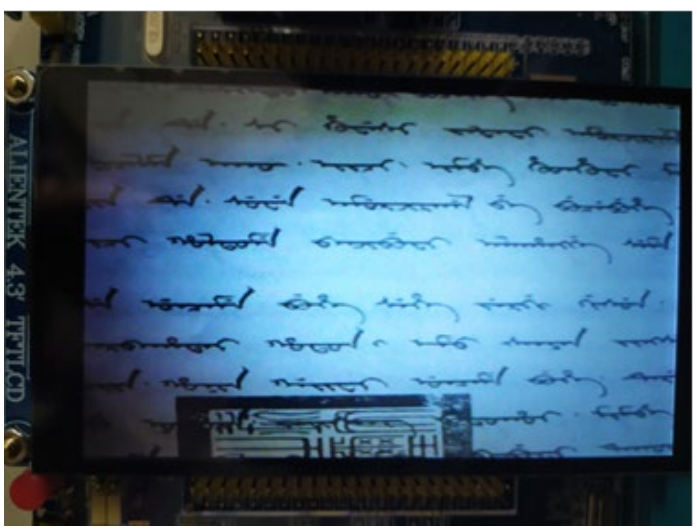

Fig.6. Manchu image acquisition and testing

After image acquisition, the image display module needs to be tested. First, the main interface of image display is shown in the Fig. below. After entering the main interface, select the image you want to view and click. Click backward to enter the mode of automatic play. Automatically play the first picture you click on. When playing automatically, click the middle area of the screen to pause the play, and click again to resume the play. Click the top and bottom of the screen to control the up and down movement ${ }^{6}$. BMP format image display is shown in the Fig. 7 below.

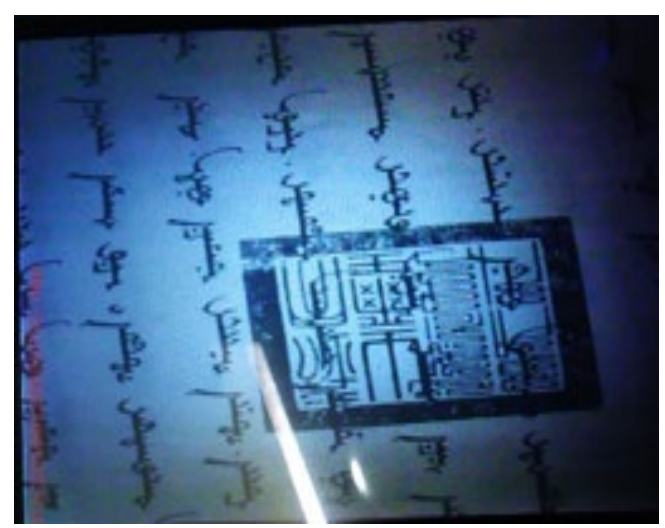

Fig.7. BMP format image display

This paper compares the advantages and disadvantages of various data transmission methods, and uses USB data cable to connect with the upper computer. According to the USB communication protocol, a kind of analog USB flash disk is realized. Click the data transmission icon, the system will enter the state of analog $U$ disk and communicate with the upper computer. Complete the system function of data transmission.

\section{References}

1. Shuang $\mathrm{Xu}, \mathrm{Min} \mathrm{Li}$, Rui-Rui Zheng, et al. Manchu character segmentation and recognition method. 2017, 20(1): 43-53.

2. M. LI, R. ZHENG, S. XU, F. WANG. Design and Implementation of Embedded Handwritten Manchu Letter Recognition System, 2018 International Conference on Information Systems and Computer Aided Education (ICISCAE), Changchun, China, 2018, pp. 7-11.

3. R. Zheng, M. Li, J. He, J. Bi, B. Wu. Segmentation-Free Multi-Font Printed Manchu Word Recognition Using Deep Convolutional Features and Data Augmentation, 2018 11th International Congress on Image and Signal Processing, BioMedical Engineering and Informatics (CISP-BMEI), Beijing, China, 2018

4. Guang-yuan Zhang, Jing-jiao Li, Rong-wei He, Ai-xia Wang. An offline recognition method of handwritten primitive Manchu characters based on strokes. Ninth International Workshop on Frontiers in Handwriting Recognition, Kokubunji, Tokyo, Japan, 2004, pp. 432-437.

5. M. Li, R. Zheng, S. Xu, Y. Fu, D. Huang. Manchu Word Recognition Based on Convolutional Neural Network with Spatial Pyramid Pooling, 2018 11th International Congress on Image and Signal Processing, BioMedical Engineering and Informatics (CISP-BMEI), Beijing, China, 2018.

6. Choi Minseok, Lee Choong Ho. A Study on the Preprocessing for Manchu-Character Recognition. Journal of the Institute of Convergence Signal Processing, 2013, 14(2). 